\title{
Problems of myeloma in a community
}

\author{
N.G. Flanagan, J.C. Ridway, A. Jain, * C.C. Platt \& A.G. Irving
}

Department of Haematology, Victoria Hospital, Blackpool, Lancashire FY3 8NR, UK.

\begin{abstract}
Summary: The presenting features of 120 consecutive cases of myeloma diagnosed in a single unit were examined. Screening tests on routine laboratory samples increased the detection rate but did not significantly reduce the percentage of cases presenting with late stage disease. Morbidity was closely related to the incidence of bone involvement already present at diagnosis. About one fifth of cases had reported suspicious symptoms for some considerable time before further investigation was forthcoming. Almost half had 'benefitted' by having concurrent disease which often led to the discovery of myeloma at an early stage. These findings, together with the wide variety of symptomatology and few physical signs, stressed the critical importance of having a high index of suspicion and thus taking suitable blood samples without unnecessary delay.
\end{abstract}

\section{Introduction}

Although the usually quoted figures for the incidence of myeloma parallel those of acute leukaemia, it is uncommon under the age of 40 years. It has therefore attracted less research interest than the leukaemias and this disease which is frequently disabling and usually fatal has, until recently, been managed with chemotherapeutic protocols introduced over 25 years ago. Since a logical staging system for myeloma was proposed by the South West Oncology Group (SWOG) ${ }^{1}$ other valuable prognostic indicators, e.g., serum beta ${ }_{2}$ microglobulin levels, have been recognized. ${ }^{2}$ These have rekindled interest in the disorder and major developments in therapy have followed. ${ }^{3-5}$ However, the routine use of staging techniques has also emphasized the critical importance of early diagnosis of myeloma.

In an attempt to explore ways of recognizing early stage disease we have reviewed the presenting features of 120 consecutive cases of myeloma diagnosed in this department in a 65 month period up to autumn 1986. This group of patients has been particularly suitable for analysis because they come from a population which is geographically isolated and all have been channelled through the same diagnostic processes. These patients have, as elsewhere, presented with a variety of symptoms and several different departments have received the initial referral for specialist opinion. Delay in diag-

Correspondence: N.G. Flanagan, F.R.C.P., F.R.C.Path. *Present address: Department of Haematology, Manchester Royal Infirmary, Oxford Road, Manchester, UK.

Accepted: 16 March 1988 nosis should have been minimized by (a) a high index of suspicion of individual clinicians, (b) screening tests on blood samples they have provided and (c) the availability of this "captive population' for follow-up confirmatory tests. The survival pattern of this group is also examined although, as many of these patients have been registered in therapeutic trials, detailed discussion of treatment is not included here.

\section{Patients and methods}

On referral, 113 patients were included in our usual investigative protocol for suspected myeloma, i.e., history, physical examination and assessment of performance status (WHO classification) which divides patients into 5 grades: Grade 0 unrestricted normal activity, Grade 1 restricted in strenuous activity, Grade 2 capable of all self care but unable to work, Grade 3 capable of limited self care partially confined to bed or chair, and Grade 4 completely disabled. The following investigations were done: full blood count (Coulter counter S plus or Technicon $\mathrm{H} 1$ ), erythrocyte sedimentation rate (ESR) and plasma viscosity, peripheral blood and marrow smears, biochemical (Technicon SMA2) and immunoglobulin profiles, serum beta, microglobulin $\left(\beta_{2} M G\right)$ (58 patients from 1.1.84) (Phadebas radioimmunoassay - Pharmacia), serum $B_{12}$ and red cell folate assays, radiographic skeletal survey and electrocardiograph. A further seven cases were too ill to be included in the full screen but a firm diagnosis of myeloma was established.

(C) The Fellowship of Postgraduate Medicine, 1988 
The diagnosis of myeloma was based on the finding of at least two of the following: (1) a homogeneous paraprotein band on serum electrophoresis and/or detection of serum or urinary light chains; (2) lytic bone lesions; (3) significant marrow plasmacytosis; usually greater than $20 \%$ plasma cells which include abnormal forms. (Sometimes the myeloma cells present as microplasmacytomas and the overall percentage infiltration in these cases is hard to assess.) Table I details the SWOG staging system for myeloma.

\section{Results}

Sixty four males and 56 females between the ages of 48 and 85 years were referred from 3 main sources, general medicine, orthopaedics and the general practitioner. Other sources included psychiatry with acute mental confusion, neurology with peripheral neuropathy, otolaryngology with deafness, ophthalmology with diplopia, dermatology with leucocytoclastic vasculitis, gynaecology with pelvic mass, and general surgery with priapism.

\section{Clinical features at presentation}

Eighty two patients complained of some form of bone pain. Of these, 63 had radiological skeletal changes which supported the diagnosis of myeloma. Established concurrent disease was found in $18(29 \%)$ of these 63 patients with bone pathology but in $41(79 \%)$ of the 57 without evidence of skeletal damage. Table II shows the pattern of

Table I Criteria for staging plasma cell myeloma patients (SWOG)

\begin{tabular}{ll}
\hline Stage & \multicolumn{1}{c}{ Criteria } \\
\hline I & All of the following: \\
& 1. Haemoglobin $>10 \mathrm{~g} / \mathrm{dl}$ \\
& 2. Normal serum calcium \\
& 3. Normal bone structure, or solitary bone \\
plasmacytoma \\
4. Low $\mathrm{M}$-protein production rates: \\
(a) $\mathrm{IgG}<5.0 \mathrm{~g} / \mathrm{dl}$ \\
(b) $\mathrm{IgA}<3.0 \mathrm{~g} / \mathrm{dl}$ \\
(c) Urinary $\kappa$ or $\lambda<4 \mathrm{~g} / 24 \mathrm{~h}$ \\
II & Fitting neither Stage I nor III \\
III & One or more of the following: \\
& 1. Haemoglobin $<8.5 \mathrm{~g} / \mathrm{dl}$ \\
& 2. Serum calcium $>3.0 \mathrm{mmol} / 1$ \\
& 3. More than 3 lytic bone lesions \\
4. High M-protein production rates: \\
(a) IgG $>7.0 \mathrm{~g} / \mathrm{dl}$ \\
(b) IgA $>5.0 \mathrm{~g} / \mathrm{dl}$ \\
(c) Urinary $\kappa$ or $\lambda>12.0 \mathrm{~g} / 24 \mathrm{~h}$
\end{tabular}

Table II Incidence of concurrent disease in 120 myeloma patients with and without bone pathology

\begin{tabular}{lcc}
\hline $\begin{array}{c}\text { Concurrent } \\
\text { disease }\end{array}$ & $\begin{array}{c}\text { With bone } \\
\text { pathology } \\
(n=63)\end{array}$ & $\begin{array}{c}\text { Without bone } \\
\text { pathology } \\
(n=57)\end{array}$ \\
\hline Cardiovascular & 8 & 12 \\
Respiratory tract & 4 & 5 \\
Connective tissue & 3 & 4 \\
Neurological & 0 & 6 \\
Endocrine & 2 & 4 \\
Genitourinary tract & 0 & 3 \\
Gastrointestinal tract & 1 & 3 \\
Solid tumour & 0 & 4 \\
\multicolumn{1}{c}{ Totals } & - & -41 \\
\hline
\end{tabular}

Table III Complications at presentation in 120 myeloma patients

\begin{tabular}{lc}
\hline \multicolumn{1}{c}{ Complications } & $\begin{array}{c}\text { No. of } \\
\text { patients } \\
(\%)\end{array}$ \\
\hline Bone pathology & $63(52.5)$ \\
Renal impairment & $23(19.2)$ \\
Hypercalcaemia $($ serum calcium $>2.8 \mathrm{mmol} / \mathrm{l})$ & $12(10.0)$ \\
Anaemia $(\mathrm{Hb}<10 \mathrm{~g} / \mathrm{dl})$ & $36(30.0)$ \\
Infection $($ respiratory/urinary) & $15(12.5)$ \\
Hyperviscosity & $6(5.0)$ \\
Spinal cord compression & $3(2.5)$ \\
Thrombotic complications & $5(4.2)$ \\
\hline
\end{tabular}

concurrent disease in the two groups. The age and sex distribution was similar in both. Other features resulted mainly from the complications listed in Table III which also illustrates the variety of clinical pictures which might be expected from a group of this size. Forty eight of the patients $(40 \%)$ were classified in stage III at diagnosis. Of these, 20 were included in the group of 24 patients where there was good evidence of significant delay ( $>3$ months) between the first reporting of suspicious symptoms and the first blood sample.

\section{Haematological findings}

Table IV compares the level of ESR with immunoglobulin class and stage at diagnosis. All 8 cases in stage I with ESR levels over 100 had concurrent disease which presumably contributed to this finding.

Significant anaemia $(<10 \mathrm{~g} / \mathrm{dl})$ without haematinic deficiency was present in almost onc third of cases yet symptoms of anaemia were notably lacking. Thrombocytopenia and leucopenia related to marrow infiltration was documented in less than $10 \%$ and contributed little to presenting symptoms. 
Table IV ESR at presentation related to clinical stage and immunoglobulin class

\begin{tabular}{|c|c|c|c|c|c|c|c|c|}
\hline \multirow[b]{2}{*}{$E S R$} & \multicolumn{2}{|c|}{ Stage } & \multirow[b]{2}{*}{ Total } & \multicolumn{5}{|c|}{ Immunoglobulin class } \\
\hline & $I$ & $I I I I I$ & & $\operatorname{Ig} G(72)$ & $\operatorname{Ig} A(38)$ & $\operatorname{Ig} M$ & $B J$ & $N S$ \\
\hline$<49$ & 13 & 40 & 17 & 15 & 0 & 0 & 1 & 1 \\
\hline 49-99 & 23 & 1011 & 44 & 24 & 15 & 1 & 3 & 1 \\
\hline$>99$ & 8 & 935 & 52 & 29 & 20 & 0 & 3 & 0 \\
\hline & - & -- & $\bar{L}$ & - & - & - & $\overline{-}$ & - \\
\hline Totals* & 44 & 2346 & 113 & 68 & 35 & 1 & 7 & 2 \\
\hline
\end{tabular}

*7 cases died prior to transfer and did not have ESR recorded.

BJ = Bence Jones; NS $=$ non-secretory.

\section{Biochemical findings}

Of the 120 cases 72 were of the IgG class, $38 \operatorname{IgA}$, 1 IgM, 7 'light chain only' and 2 non-secretory. The overall kappa:lambda ratio was $2: 1$ and was without significant variation within the immunoglobulin classes. Immunoglobulin class was compared with presenting ESR level (113 patients). Significant association was only found in 17 cases with an ESR of less than $49 \mathrm{~mm}$ in one hour where none were of the IgA class.

Uric acid levels were elevated in 17 cases; 9 of these could be explained by renal impairment of concurrent diuretic therapy.

Although abnormality of renal function was found frequently at presentation, only 23 cases showed persistent impairment after initial rehydration. Half the 12 patients with hypercalcaemia had classical symptoms including acute mental confusion, polydypsia, polyuria and constipation.

Thirty seven of the 58 patients on whom $\beta_{2}$ MG levels were obtained at diagnosis had a level in excess of $3 \mathrm{mg} / \mathrm{l}$. Like Childs et al. ${ }^{6}$ we selected this as the upper normal level in this age group. Only 4 of these were Stage I and 22 of the remainder were Stage III thus showing good correlation between $\beta_{2}$ MG levels and stage of disease. However this quite sophisticated test is not appropriate as a routine screening procedure.
Table VI Effect of pre-existing bone damage on performance status (WHO classification) at presentation

\begin{tabular}{lcccccc}
\hline & \multicolumn{5}{c}{ Performance status } & \\
\cline { 2 - 6 } & 0 & 1 & 2 & 3 & 4 & Total \\
\hline No bone & 14 & 15 & 15 & 10 & 3 & 57 \\
damage & $(25 \%)$ & $(27 \%)$ & $(27 \%)$ & $(17 \%)$ & $(5 \%)$ & $(48 \%)$ \\
Bone & 0 & 11 & 27 & 20 & 5 & 63 \\
damage & $(0 \%)$ & $(17 \%)$ & $(43 \%)$ & $(32 \%)$ & $(8 \%)$ & $(52 \%)$ \\
\hline
\end{tabular}

One year after completion of the study 37 patients are still alive. Table $\mathrm{V}$ lists some details of the other 83 patients. Forty two died within the first year and of these only 13 were considered suitable for specific chemotherapy. Advanced age, other serious disease and end-stage myeloma at diagnosis precluded therapy in the others. Complications of myeloma causing death were almost all infective or renal.

Table VI compares the performance status of patients with and without bone damage at presentation. As a group those without bone lesions had significantly less morbidity throughout their illness.

\section{Discussion}

The problems of myeloma in a community are well illustrated on the Fylde Coast. The population of 310,000 here is geographically isolated by the Irish Sea to the north and west, the Ribble estuary to the south and a motorway system on the eastern border. Myeloma is a disease par excellence where delay in diagnosis can greatly increase morbidity and influence survival because of the development of its typical complications particularly bone damage. Many patients in this 'captive' population have considerable distances to travel to the only general hospital and the disability caused by myeloma involving bone has been distressing to the individual and costly for the Health Authority. Skeletal damage has had the greatest adverse effect

Table V Survival time and cause of death related to treatment and stage at presentation

\begin{tabular}{|c|c|c|c|c|c|c|c|c|}
\hline \multirow{3}{*}{$\begin{array}{l}\text { Survival } \\
\text { (months) }\end{array}$} & \multirow[b]{3}{*}{ Died } & \multicolumn{3}{|c|}{ Cause of death } & \multirow{3}{*}{$\begin{array}{l}\text { Number } \\
\text { treated }\end{array}$} & \multirow{2}{*}{\multicolumn{3}{|c|}{ Stage }} \\
\hline & & & \multirow{2}{*}{$\begin{array}{c}\text { Myeloma } \\
\text { complications }\end{array}$} & \multirow{2}{*}{$\begin{array}{c}\text { Unrelated } \\
\text { disease }\end{array}$} & & & & \\
\hline & & Myeloma & & & & $I$ & $I I$ & III \\
\hline$<6$ & 33 & 4 & 13 & 16 & 5 & 11 & 7 & 15 \\
\hline $6-12$ & 9 & 4 & 2 & 3 & 8 & 3 & 0 & 6 \\
\hline $12-24$ & 19 & 5 & 9 & 5 & 17 & 5 & 4 & 10 \\
\hline $24-36$ & 12 & 6 & 6 & 0 & 12 & 5 & 5 & 2 \\
\hline over 36 & 10 & 5 & 5 & 0 & 10 & 2 & 3 & 5 \\
\hline Totals & 83 & 24 & 35 & 24 & 52 & 26 & 19 & 38 \\
\hline
\end{tabular}


on morbidity because although chemotherapy can usually reduce the tumour load, pre-existing bone lesions may delay a satisfactory clinical response and lead to frequent periods of expensive in-patient management.

In an attempt to detect the disease at an early stage a screening programme was introduced in this department in 1983. This has involved (a) electrophoresis of all serum samples with globulin levels over $35 \mathrm{~g} / 1$ irrespective of the source of the samples or the provisional diagnosis and (b) marrow examination and electrophoresis at an early stage in patients with an unexplained persistently raised ESR. This screening procedure has, of course, revealed a considerable number of patients with paraproteinaemia who were excluded from this investigation because they did not fulfill the criteria for a diagnosis of myeloma.

In an earlier study 79 cases of myeloma were diagnosed here in a 5-year period. The expected, age corrected, figure was 79.8. In this latest study, 120 cases have been observed in a 65 -month period with an expected figure of 86.3 (calculations based on cancer registration for England and Wales 197780). It seems likely that this apparent increase can be mainly attributed to the use of screening tests for most of the second study rather than a genuine excess of cases and supports our contention that myeloma is regularly under reported. In particular, the screening procedure has led to the diagnosis of myeloma in 7 patients who died fairly soon after emergency admission and would otherwise have been labelled as cardiac or respiratory problems.

It has been disappointing therefore to find that the number of cases seen first with late stage disease is much the same in the two studies, i.e., around $40 \%$. Unfortunately about one fifth of the patients had reported symptoms suggestive of serious disease yet had suffered delays of more than 3 months before further investigation. All but 4 of these were in stage III at diagnosis. Indeed on the evidence of this study a case could almost be made that a patient with unsuspected myeloma might fare better if he had concurrent disease which generated early blood sampling.

Examination of the mode of presentation of these patients emphasizes the difficulty of early detection but offers some hope of improvement. Because useful clinical signs tend to be minimal in patients with myeloma it is very important that the first examining physician has a high index of suspicion and organizes blood samples or specialist referral at an early date. Myeloma-induced bone pain is all too often described as 'arthritis' by the patient especially if elderly. A careful history, sometimes admittedly taken in retrospect after the diagnosis had been suspected, was usually sufficient to dis- tinguish between bone and joint pain. Myelomainduced pain was frequently reported as only occurring with movement and not disturbing sleep; this proved a most helpful feature. Bone damage in unusual sites, such as the thoracic spine or sternum, without a history of injury, or osteoporosis in the 'wrong age group' were suspicious radiological signs even in the absence of typical myeloma changes. We believe that if symptoms justify skeletal X-rays, blood samples should be taken at the same time.

Although infection was not a very prominent presenting feature in this series some patients had had repeated episodes. Where cultures had been taken, Escherichia coli and Streptococcus pneumoniae were the commonest organisms found. Most of these episodes had responded to simple antibiotic therapy thus allaying suspicion. However, the repeated pattern of infection demanded further investigation. In general the other myeloma complications seen at presentation had led to specialist referral at an early date and usually to a diagnosis soon after.

The screening procedure had its own problems. Although it was possible to spread the investigations evenly to avoid a significant extra laboratory work load, all non-myeloma paraproteinaemias had to be investigated further. We decided that a total globulin of $35 \mathrm{~g} / \mathrm{l}$ was the most useful level at which to initiate electrophoresis of routine samples. Some patients with IgA myeloma may have significant levels of paraprotein which are hidden within a normal globulin. Lowering the cut off level of $35 \mathrm{~g} / \mathrm{l}$ may detect these cases but greatly increases the numbers of non-myeloma paraproteinaemias.

In our experience when samples are first taken they are very much more likely to include a request for ESR than biochemistry. In this study a level of $49 \mathrm{~mm}$ fall in 1 hour was regarded as significantly raised for screening purposes. Eighteen percent would have been missed on this information alone although none were $\operatorname{IgA}$ class. We believe this is a useful screening test when serial readings are made although it has many limitations. Plasma viscosity measurements were unsuitable for this type of screening programme but, like beta ${ }_{2}$ microglobulin levels, were very useful as part of follow up investigations.

Many studies of myeloma survival figures refer only to patients who have had specific treatment. It was interesting therefore to note the effect that deaths of untreated patients had on the overall figures. Twenty eight of the 33 patients who died within 6 months were not given specific treatment because of other serious disease, general frailty or end-stage myeloma. Age alone did not preclude therapy. It was deferred in those who had presented 
with early stage disease until there was evidence of progression. Response to treatment and survival statistics in this group with a wide age range was much as expected when compared to published figures. ${ }^{8}$

Morbidity was difficult to assess quantitatively but in retrospect a record of performance status at presentation had provided a helpful guide to the likelihood of subsequent complications. Pre-existing bony injury was shown clearly to have a detrimental effect on this assessment, as indicated in the Third Working Party report on Leukaemia in Adults. ${ }^{9}$ Restriction of movement caused by pain predisposed to respiratory and urinary infection with regular hospital admission. At the time of writing patients with myeloma bone damage occupy about $10 \%$ of our oncological work load yet are responsible for over $50 \%$ of non-routine outpatient

\section{References}

1. Durie, B.G.M. \& Salmon, S.E. A clinical staging system for multiple myeloma. Cancer 1975, 36: 842-854.

2. Cusick, J., Cooper, E.H. \& MacLennan, I.C.M. The prognostic value of serum beta ${ }_{2}$ microglobulin compared with other presentation features in myelomatosis. Br J Cancer 1985, 52: 1-6.

3. Malpas, J.S. New treatments in myeloma - is cure possible? Postgrad Med J 1987, 63: 425-426.

4. Barlogie, B., Smith, I. \& Alexanian, R. Effective treatment of advanced multiple myeloma refractory to alkylating agents. $N$ Engl J Med 1984, 310: 1353-1357.

5. McIlwain, T.M., The modern management of myeloma. In: Pounder, R.E. \& Chiodini, P.L. (eds) Advanced Medicine 23, Bailliere Tindall, London, 1987, pp 215-230. appointments. We have no doubt that any attempt to detect the disease before skeletal damage is to be welcomed. In spite of the number of cases presenting here with late stage disease we firmly believe that screening tests of the type described make a valuable contribution to early diagnosis. It is worth noting that myeloma would have been suspected in 114 cases on the basis of the screening tests alone. However, we emphasize that any beneficial effect that these tests have on morbidity is entirely dependent on early sampling in order to influence morbidity.

\section{Acknowledgements}

The authors greatly appreciate the support provided by the Department of Chemical Pathology.

6. Child, J.A., Crawford, S.M., Norfolk, D.R., O'Quigley, J., Scarffe, J.H. \& Struthers, L.P.L. Evaluation of serum Beta ${ }_{2}$ microglobulin as a prognostic indicator in myelomatosis. $\mathrm{Br} J$ Cancer 1983, 47: 11-114.

7. Flanagan, N.G., Harry, D.S., Kozlowski, C.L. \& Sorahan, T. Multiple myeloma on the Fylde coast. $\mathrm{Br}$ Med J 1984, 289: 1075.

8. Bataille, R., Durie, G.M., Grenier, J. \& Sany, J. Prognostic factors and staging in multiple myeloma: a reappraisal. J Clin Oncol 1984, 4: 80-87.

9. MRC Working Party on Leukaemia in Adults. Prognostic features in the third myelomatosis trial. $\mathrm{Br}$ $J$ Cancer 1980, 42: 831-840. 